\title{
Metacercarias de la familia Microphallidae (Trematoda: Digenea) en el anfípodo Hyale grandicornis en la costa de Maule, Chile Central
}

\author{
GABRIELA MUÑOZ*
}

\author{
METACERCARIAE OF THE FAMILY MICROPHALLIDAE (TREMATODA: DIGENEA) IN THE \\ AMPHIPOD Hyale grandicornis FROM THE COAST OF MAULE, CENTRAL CHILE
}

Digeneans have complex life cycles with 2 reproductive phases and 2-3 hosts such as molluscs, crustaceans and vertebrates. Despite numerous records of adult digeneans from many host species of Chile, there are few records in invertebrates. The aim of this study is to provide information about juvenile phases of digeneans in the amphipod Hyale grandicornis (Kröyer, 1845). Ninety-eight amphipods were collected from intertidal pools at Maule, central coast of Chile. Sixty-four percent of the amphipods were parasitized with 1-25 metacercariae/host individual. Three types metacercariae were distinguished according their shape (spherical or ovalated) and size (between 200 to $375 \mu \mathrm{m}$ legth). To identify them, the metacercariae were incubated and artificially opened. The morphology of juvenile digeneans of the 3 types of metacercariae corresponded to the family Microphallidae. One type of metacercaria may belong to Maritrematinae; the other two may belong to Microphallinae. The definitive hosts for Microphallidae are principally birds, although some species can be found in fishes, amphibians or mammals. In Chile, there are no records of these digeneans in any group of hosts. In the Coast of Maule usually inhabit many bird species such as Larus, Fulica, and Phalacrocorax genera, so that some of them could be definitive hosts of these digeneans.

Key words: Microphallidae, metacercariae, amphipods, Hyale, Chile.

\section{INTRODUCCIÓN}

Los trematodos digenéticos son un grupo de parásitos muy numeroso y diverso. Se caracterizan por su complejo ciclo de vida el cual consta de dos fases reproductivas y necesitan de al menos 2 hospedadores para lograr su fase adulta ${ }^{1}$. El primer hospedador intermediario es un molusco y el segundo usualmente es un crustáceo, aunque existen otras alternativas para sus ciclos de vida.
Los hospedadores definitivos son vertebrados como peces, anfibios, aves o mamíferos ${ }^{1}$.

En Chile, se han registrado cercarias de digeneos en caracoles ${ }^{2}$ y en bivalvos ${ }^{3}$, metacercarias $^{4}$ y metacercarias progenéticas en gastró$\operatorname{podos}^{5-6}$. Existen numerosos registros de digeneos adultos en distintas especies de peces litorales ${ }^{7-8}$, algunos en aves ${ }^{9-12}$, y muy pocos en mamíferos marinos ${ }^{13}$. Sin embargo, no hay registros de alguna fase de digeneos en crustáceos.

School of Molecular and Microbial Sciences, Department of Microbiology and Parasitology, University of Queensland, Brisbane 4072, Qld, Australia. e-mail: s4005684@ student.uq.edu.au 
En la zona del intermareal habitan numerosas especies de peces y aves que presentan variadas tramas tróficas. Sus dietas incluyen usualmente a pequeños crustáceos como isópodos, anfípodos, decápodos y peces pequeños ${ }^{14-16}$. Por lo tanto, es posible esperar que fases larvales de digeneos vivan en estos ítems dietarios, llegando a sus hospedadores definitivos por vía trófica.

El presente estudio tuvo como objetivo revisar la presencia de digeneos en una especie de "pulga de mar", que son anfípodos que habitan en la zona intermareal de Chile.

\section{MATERIAL Y MÉTODOS}

Noventa y ocho anfípodos fueron obtenidos desde posas intermareales expuestas cerca de Caleta Maule, Chile ( $\left.37^{\circ} 30^{\prime} \mathrm{S} ; 7^{\circ} 14^{\prime} \mathrm{O}\right)$, mediante redes de mano en el verano de 2001. Todos los ejemplare eran de la especie Hyale grandicornis (Kröyer, 1845) los cuales fueron identificados siguiendo a González (1991) ${ }^{17}$. Estos fueron mantenidos vivos hasta el momento de su disección. La longitud total de los anfípodos fue medida con un pie de metro de $0,1 \mathrm{~mm}$ de sensibilidad.

La disección de los anfípodos se realizó bajo un microscopio estereoscópico y consistió en colocar al crustáceo en una placa petri con agua de mar diluída (25\%), luego cada segmento del anfípodo fue abierto con pinzas de punta fina. $\mathrm{Si}$ había metacerarias estas quedaban libres en el agua de la placa. Se registró el número de metacercarias por individuo y cada una de ellas fue medida en su largo y su ancho mediante un reglilla de ocular acoplado a un microscopio óptico. Para distinguir tipos de metacercarias se observó su morfología (tamaño y forma). Luego, para determinarlas taxonómicamente, se procedió a incubar las metacercarias y hacerlas eclosionar. Con este propósito las metacercarias fueron sumergidas en suero fisiológico con sales biliares de cerdo (Desicol $\left.{ }^{\circledR}\right)$. Posteriormente, fueron puestas a temperatura del ambiente de laboratorio (20-25 $\mathrm{C})$, que sería similar a la temperatura natural de donde fueron capturados. Los digeneos obtenidos fueron observados al natural bajo microscopio óptico.

\section{RESULTADOS Y DISCUSIÓN}

La longitud total de los anfípodos tuvo un promedio de $10,7 \pm 2,6 \mathrm{~mm}$. El $64,3 \%$ de los anfípodos albergaba entre 1 y 25 metacercarias. No se encontraron estado de esporoquistes, tampoco otro tipo de parásitos. Morfológica y métricamente se distinguieron 3 tipos de metacercarias: una de forma ovalada cuyas medidas fueron entre $280-350 \mu \mathrm{m}$ de largo y 200 $290 \mu \mathrm{m}$ de ancho (tipo 1, Figura 1A); otras de forma esféricas de 300-350 $\mu \mathrm{m}$ (tipo 2, Figura 1B) que presentaba una cubierta gruesa; y otras ligeramente más grandes de 350-375 $\mu \mathrm{m}$ de diámetro, con una cubierta más delgada que la anterior (tipo 3, Figura 1C). La más abundante y prevalente fue la de tipo 1 (Tabla 1). En la mayoría de los anfípodos se encontró un tipo de metacercaria, en 7 se encontraron 2 tipos y en sólo 2 ejemplares se registraron los 3 tipos de metacercarias.

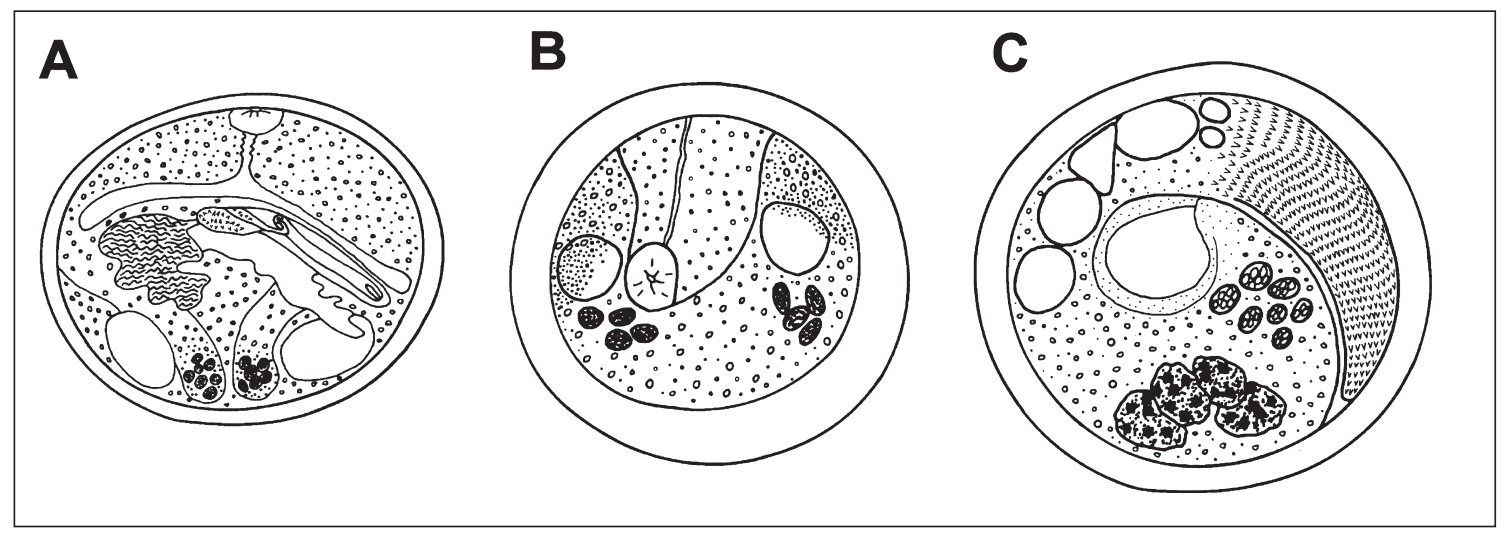

Figura 1. Morfología de 3 tipos de metacercarias de Microphallidae encontradas en el anfípodo Hyale grandicornis. A) tipo 1, B) tipo 2 y C) tipo 3. Línea de cota $=100 \mu \mathrm{m}$. 


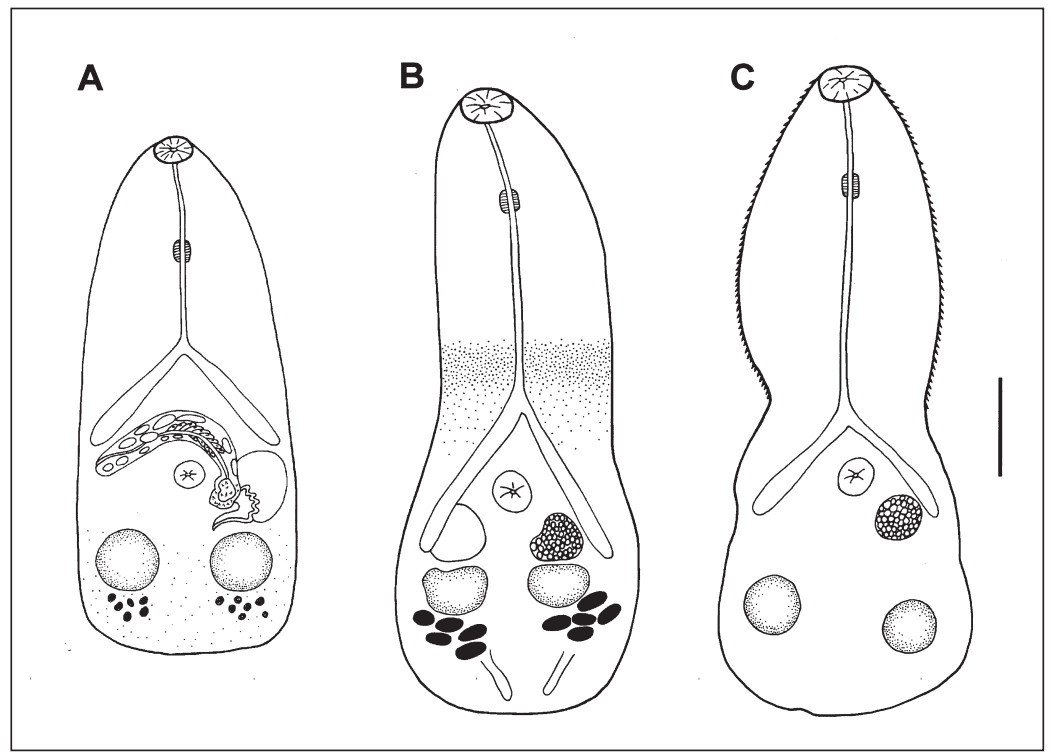

Figura 2. Morfología de los digeneos juveniles provenientes de cada tipo de metacercaria. A) tipo 1, B) tipo 2 y C) tipo 3. Línea de cota $=100 \mu \mathrm{m}$.

Ciento treinta metacercarias tipo 1 fueron incubadas, de las cuales 40 eclosionaron entre las 2 y las 72 horas después de colocarlas en un medio artificial (Tabla 1). En cambio, las metacercarias tipo 2 y 3 que fueron incubadas, ninguna eclosionó exitosamente. Sólo algunas de ellas fueron abiertas manualmente con bisturí y pinzas.

La morfología de los digeneos juveniles de los 3 tipos de metacercarias corresponde a la familia Microphallidae. Aquellos individuos provenientes de las metacercarias tipo 1, fueron ligeramente mas pequeños que los otros tipos (Tabla 1). Este tipo de metacercaria se caracterizó por el saco del cirro semicurvo en ubicación preacetabular en la mitad del cuerpo del digeneo, atrio genital espinado, algunas vitelógenas localizadas posterior a las testis (Figura 2A). Estos digeneos pertenecerían a Maritrematinae (pers. com. Dr. Deblock), aunque en este grupo la distribución de las vitelógenas es semicircular a circular, como un anillo en la mitad posterior del cuerpo. Esta distribución de las vitelógenas podría ser vista en estado de metacercaria ${ }^{18}$, pero difiere de lo observado con los ejemplares del presente estudio (Figuras 1A, 2A). Tampoco se observaron espinas corporales que son comunes in Microphallidae $^{19}$. Las variaciones encontradas en estas metacercarias podrían deberse al temprano estado de desarrollo de estos parásitos y a la incubación artificial de estos. Un análisis basado en incubación de los digeneos por más tiempo permitiría confirmar la consistencia de estas características morfológicas.

A los digeneos juveniles tipo 2, no se les observaron espinas corporales (quizás eran muy pequeñas o no estaban desarrolladas), y tienen su cuerpo más ancho en la parte posterior. Los folículos de vitelógenas son medianamente grandes y posteriores a las testis. No se les distinguió saco del cirro (Figura 2B). El digeneo juvenil tipo 3, fue el más difícil de obtener manualmente desde la metacercaria. Aparentemente, en la mitad del cuerpo hay una constricción; la mitad anterior del cuerpo es muy espinoso (Figura 2C); espinas grandes y visibles en el estado de metacercaria (Figura 1C). Vitelógenas no vistas en el digeneo juvenil aunque en la metacercaria se observaron cuerpos vitológenos medianamente grandes (Figura 1C). Los digeneos tipo 2 y 3 podrían pertenecer a Microphallinae, principalmente por la ausencia del saco del cirro ${ }^{19}$.

Los hospedadores definitivos de Maritrematinae son aves y algunos mamíferos, mientras que en Microphallinae pueden ser peces, aves, anfíbios y mamíferos ${ }^{19}$. En Chile, no hay registros de estos digeneos en aves ${ }^{9-12}$, ni en ningún otro 
Tabla 1. Número total de metacercarias (n) obtenidas de la muestra de anfípodos, prevalencia (P), abundancia y su desviación estándar (abun \pm d.e.); número de metacercarias incubadas y eclosionadas, y el rango de tamaño de los digeneos juveniles $(e n \mu \mathrm{m})$ proveniente de cada tipo de metacercaria

\begin{tabular}{lrrrrrr}
\hline Metacercaria & n & P(\%) & Abun \pm d.e. & $\begin{array}{c}\mathbf{N}^{\mathbf{0}} \\
\text { incubadas }\end{array}$ & $\begin{array}{c}\mathbf{N}^{\mathbf{2}} \\
\text { eclosionadas }\end{array}$ & $\begin{array}{c}\text { Rango de tamaño } \\
\text { largox ancho }\end{array}$ \\
\hline Tipo 1 & 274 & 61,2 & $2,8 \pm 4,0$ & 130 & 40 & $360-520 \times 180-300$ \\
Tipo 2 & 10 & 6.1 & $0,1 \pm 0,5$ & 10 & $3 *$ & $600-640 \times 250-270$ \\
Tipo 3 & 18 & 6,1 & $0,2 \pm 0,9$ & 15 & $2 *$ & $630-715 \times 260-270$ \\
Total & 302 & & $3,1 \pm 4,4$ & 155 & 45 & - \\
\hline
\end{tabular}

*eclosionadas manualmente.

grupo de vertebrado. Debido a que los anfípodos habitaban en pozas intermareales expuestas, los depredadores de estos crustáceos podrían ser principalmente aves, por lo tanto estos podrían ser los hospedadores definitivos para estos digeneos microfálidos. En el ambiente en donde se obtuvieron las muestras, se encuentran comunmente gaviotas (Larus), taguas (Fulica) y cormoranes (Phalacrocorax). Hay registros de especies de Microphallidae en Larus spp. de sistemas lacustres de Argentina ${ }^{18,20}$. Sin embargo, varias otras especies de aves habitan temporalmente la costa de Maule ${ }^{21}$ podrían ser los hospedadores potenciales para estos digeneos.

\section{RESUMEN}

Los digeneos presentan ciclos biológicos complejos con 2 fases reproductivas y varios hospedadores, como moluscos y crustáceos (hospedadores intermediarios) y vertebrados (hospedadores definitivos). A pesar de los numerosos registros de digeneos en fases adultas en distintas especies hospedadoras, hay muy pocos registros en invertebrados y ninguno en crustáceos. Con el fin de aportar información sobre alguna de la fases juveniles de digeneos, se revisaron 98 anfípodos Hyale grandicornis (Kröyer, 1845) recolectados desde pozas intermareales en la costa de Maule, Chile Central. De estos el 64,3\% albergaban entre 1 y 25 metacercarias por amfípodo. Se reconocieron 3 tipos de metacercarias de acuerdo a su forma (ovalada y esféricas) y tamaño (entre 200 y 375 $\mu \mathrm{m})$. Para identificarlas, las metacercarias fueron incubadas y eclosionadas artificialmente. La morfología de los digeneos juveniles corresponde a Microphallidae: un tipo correspondería a Maritrematinae, y los otros dos podrían corresponder a Microphallinae. Estos digeneos principalmente son parásitos de aves y algunos son de peces, anfibios o mamíferos, sin embargo no hay registros de estos parásitos en ningun vertebrado en Chile. Varias especies de aves como Larus, Fulica y Phalacrocorax que habitan comúnmente en las costas de Maule, podrían ser las hospedadoras definitivas de estos digeneos.

\section{REFERENCIAS}

1.- WILLIAMS H, JONES A. Parasitic worms of fish. Taylor \& Francis Ltd., London. 1994, 593 pp.

2.- OLMOS V, GEORGE-NASCIMENTO M. El gremio de larvas de Digenea en el caracol del sur de Chile Chilina dombeyana ¿qué indica la tasa metabólica de los hospedadores parasitados? Rev Chil Hist Nat 1997; 70: $109-18$.

3.- FRANJOLA R, GALLARDO C S. Estados larvales de Digenea en Kingiela chilenica (Mollusca, Bivalvia, Cyamiidae). Bol Chil Parasitol 1991; 46: 34-5.

4.- OLIVA O, OLIVARES A N, DÍAZ C D, PASTE M V. Parasitic castration in Concholepas concholepas (Gastropoda: Muricidae) due to a larval digenean in northern Chile. Dis Aquat Org 1999; 36: 61-5.

5.- GEORGE-NASCIMENTO M, BALBOA L, ALDANA M, OLMOS V. Las lapas Fissurella spp. (Mollusca: Archaeogastropoda) y el pejesapo Sicyases sanguineus (Pisces: Gobiesocidae) son hospedadores secuenciales de Proctoeces lintoni (Digenera: Fellodistomidae) en Chile. Rev Chil Hist Nat 1998; 71: 169-76.

6.- OLIVA M, HUAQUIN L G. Progenesis in Proctoeces lintoni (Fellodistomidae), a parasite of Fissurella crassa (Archaeogastropoda) in a latitudinal gradient in the Pacific Coast of South America. J Parasitol 2000; 86: 768-72.

7.- INZUNZA A, TORRES P, FRANJOLA R. Nuevos hospedadores para Helicometrina nimia Linton, 1910 (Digenea: Opecoelidae) en la costa centro sur de Chile. Bol Chil Parasitol 1989; 44: 41-3.

8.- LUQUE J, OLIVA M. Trematodes of marine fishes from the Peruvian faunistic province (Peru and Chile), with description of Lecithochirium callaoensis $\mathrm{n}$. sp. and new records. Rev Biol Mar 1993; 28: 271-86.

9.- TORRES P, FIGUEROA L, SALDIVIA A, 
BARRIENTOS J. Gastrointestinal helminths of fisheating birds from the Valdivia River, Chile. J Parasit 1982; 68: 1157.

10.- TORRES P, FIGUEROA L, SALDIVIA A. Stephanoprora denticulata (Trematoda, Echinostomatidae) en gaviotas del sur de Chile. Bol Chil Parasitol 1983; 38: $33-4$

11.- TORRES P, RUIZ E, GESCHE W, MONTEFUSCO A. Gastrointestinal helminths of fish-eating birds from Chiloe Island, Chile. J Wild Dis 1991; 27: 178-9.

12.- TORRES P, CONTRERAS A, CUBILLOS V, et al. Parasitismo en peces, aves piscívoras y comunidades humanas rivereñas de los lagos Yelcho y Tagua Tagua, X región de Chile. Arch Med Vet 1992; 24: 77-93.

13.- FERNÁNDEZ J C. Nuevos registros de parásitos en mamíferos marinos chilenos. Parasitol al Día 1987; 11: $120-6$.

14.- MUÑOZ A A, OJEDA F P. Feeding guild structure of a rocky intertidal fish assemblage in central Chile. Environ Biol fishes 1997; 49: 471-9.

15.- MUÑOZ A A, OJEDA F P. Guild structure of carnivorous intertidal fishes of Chilean coast: implications of ontogenetic dietary shifts. Oecologia 1998; 114: 563-73.

16.- GISPERT, C. Aves. Guías Visuales Océano. Océano
Grupo Editorial S. A. Barcelona, España. 1999, 318 pp.

17.- GONZÁLEZ E. The genus Hyale in Chile. Spixiana 1991; 14: 125-42.

18.- ETCHEGOIN J A, MARTORELLI S R. Description of a new species of Maritrema (Digenea: Microphallidae) from Mar Chiquita Coastal Lagoon (Buenos Aires, Argentina) with notes of its life cycle. J Parasitol 1997; 83: 709-13.

19.- YAMAGUTI S. Synopsis of digenetic trematodes of vertebrates. Keigaku Publishing Co. Tokyo, Japan. 1971; 3: 1-1074.

20.- ETCHEGOIN J A, MARTORELLI S R, SARDELLA N H. Nuevo registro de Microcephallus szidati Martorelli, 1986 (Digenea: Microphallidae) en Mar Chiquita (Buenos Aires, Argentina). Neotropica 1996; 42: $117-8$.

21.- ARAYA B, MILLIE G. Guía de campo de las aves de Chile. Editorial Universitaria. Octava Edición. 1998, 406 pp.

Agradecimientos: Se agradece al Dr. Stephane Deblock (Laboratoire de Parasitologie, Faculte de Pharmacie, Lille Cedex, France) por su opinión en la identificación de los digeneos.

ANUNCIO

PARASITOLOGIA LATINOAMERICANA EN INTERNET

InformaciÛn sobre Parasitologla Latinoamericana y su versiÛn electrÛnica se puede ahora encontrar en Internet. DirecciÛn: www.scielo.cl 